\title{
openheart \\ MRI using ultrasmall superparamagnetic particles of iron oxide in patients under surveillance for abdominal aortic aneurysms to predict rupture or surgical repair: MRI for abdominal aortic aneurysms to predict rupture or surgery - the $M A^{3} R S$ study
}

Olivia M B McBride, ${ }^{1}$ Colin Berry, ${ }^{2}$ Paul Burns, ${ }^{1}$ Roderick T A Chalmers, ${ }^{1}$ Barry Doyle, ${ }^{1}$ Rachael Forsythe, ${ }^{1}$ O James Garden, ${ }^{1}$ Kirsteen Goodman, ${ }^{1}$ Catriona Graham, ${ }^{1}$ Peter Hoskins, ${ }^{1}$ Richard Holdsworth, ${ }^{3}$ Thomas J MacGillivray, ${ }^{1}$ Graham McKillop, ${ }^{1}$ Gordon Murray, ${ }^{1}$ Katherine Oatey, ${ }^{1}$ Jennifer M J Robson, ${ }^{2}$ Giles Roditi, ${ }^{2}$ Scott Semple, ${ }^{1}$ Wesley Stuart, ${ }^{2}$ Edwin J R van Beek, ${ }^{1}$ Alex Vesey, ${ }^{1}$ David E Newby ${ }^{1}$

To cite: McBride OMB, Berry C, Burns P, et al. MRI using ultrasmall superparamagnetic particles of iron oxide in patients under surveillance for abdominal aortic aneurysms to predict rupture or surgical repair: MRI for abdominal aortic aneurysms to predict rupture or surgery-the $M A^{3} R S$ study. Open Heart 2015;2:e000190. doi:10.1136/openhrt-2014000190

- Additional material is available. To view please visit the journal (http://dx.doi.org/ 10.1136/openhrt-2014000190)

Received 7 October 2014 Accepted 18 January 2015

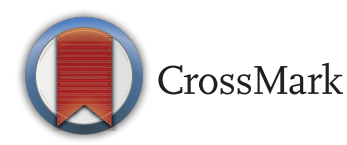

For numbered affiliations see end of article.

Correspondence to Dr Olivia M B McBride; olivia.mcbride@ed.ac.uk

\section{ABSTRACT}

Introduction: Population screening for abdominal aortic aneurysms (AAA) halves the associated mortality and has led to the establishment of national screening programmes. Prediction of aneurysm growth and rupture is challenging and currently relies on serial diameter measurements with ultrasound. Recently, a novel MRI-based technique using ultrasmall superparamagnetic particles of iron oxide (USPIO) has demonstrated considerable promise as a method of identifying aneurysm inflammation and expansion. Methods and analysis: The MA ${ }^{3} R S$ study is a prospective observational multicentre cohort study of 350 patients with AAA in three centres across Scotland. All participants will undergo MRI with USPIO and aneurysm expansion will be measured over 2 years with $\mathrm{CT}$ in addition to standard clinical ultrasound surveillance. The relationship between mural USPIO uptake and subsequent clinical outcomes, including expansion, rupture and repair, will be evaluated and used to determine whether the technique augments standard risk prediction markers. To ensure adequate sensitivity to answer the primary question, we need to observe 130 events (composite of rupture or repair) with an estimated event rate of $41 \%$ over 2 years of follow-up. The MA ${ }^{3} \mathrm{RS}$ study is currently recruiting and expects to report in 2017.

Discussion: This is the first study to evaluate the use of USPIO-enhanced MRI to provide additional information to aid risk prediction models in patients with AAA. If successful, this study will lay the foundation for a large randomised controlled trial targeted at applying this technique to determine clinical management.

\section{KEY MESSAGES}

What is already known about this subject?

- Current techniques for estimation of rupture risk in abdominal aortic aneurysm disease rely solely on diameter measurements from ultrasound However, the absolute diameter of the aneurysm is not the sole determinant of the risk of rupture.

- USPIO-enhanced MRI can be used to detect focal hotspots of inflammation in asymptomatic aneurysms, and mural USPIO uptake is associated with more rapid expansion of aneurysms.

What does this study add?

- The aim of the $M A^{3} R S$ study is to examine the relationship between mural USPIO uptake and subsequent clinical outcomes.

How might this impact on clinical practice?

- This technique may provide an improved method of risk stratification of patients with abdominal aortic aneurysms that extends beyond simple anatomical measurements of aneurysm diameter.

Trial registration number: Current Controlled Trials: ISRCTN76413758.

\section{INTRODUCTION}

Abdominal aortic aneurysm (AAA) disease is particularly prevalent in elderly men, 
affecting $5 \%$ of men over the age of 65 years $^{1}$ and causing $2-3 \%$ of deaths within this group. ${ }^{2}$ Aneurysm rupture is associated with a mortality rate in excess of $80 \% .^{3}{ }^{4}$ Population screening halves the mortality associated with AAA and has led to the establishment of national screening and surveillance programmes. ${ }^{1}$ However, once an aneurysm has been diagnosed, there is currently no accurate method of predicting which patients are at risk of rupture and would benefit from elective surgical or endovascular intervention.

The aetiology of AAA is multifactorial, with environmental and genetic factors playing a part. Aneurysms typically occur in patients with atherosclerosis and there are a number of common risk factors, however, there are also distinct differences between the two disease processes. Atherosclerotic lesions are predominantly located within the tunica intima, whereas aneurysm disease affects the media and also the adventitia. Aneurysm disease is most commonly confined to the infrarenal aorta, whereas atherosclerosis is generally more widespread throughout the arterial tree. In aneurysm disease, cigarette smoking and hypertension are more strongly correlated with incidence and risk of rupture. ${ }^{5}$

The combined effects of biomechanical factors and biological processes weaken the aortic wall, and lead to aneurysm formation, expansion and rupture. Histopathologically, the aneurysmal aortic wall is characterised by focal medial neovascularisation, infiltration of inflammatory cells (principally macrophages) and fragmentation of elastin and collagen fibres within the extracellular matrix. Regions of intense biological activity represent sites of potential rupture, and can be considered as putative targets for novel imaging techniques to predict aneurysm expansion and assess the risk of rupture. In addition, tissue and wall stresses vary spatially within the aneurysm, and tensile strength varies in different parts of the aneurysm sac. ${ }^{6}$ It is likely that expansion and rupture occur where regions of high biomechanical stress coincide with these biological 'hotspots' in the aneurysm wall that have become weakened. Indeed, there is strong evidence that aneurysm rupture is seen in those patients with more rapid aneurysm expansion rates. $^{89}$

\section{MRI}

MRI is increasingly being used as an investigative tool for cardiovascular disease, enabling distinction between the different atherosclerotic plaque components, such as the lipid-rich necrotic core, fibrous cap and areas of calcification. ${ }^{10}$ Standard gadolinium-based MRI is able to identify areas of thrombus formation and fibrosis in AAA. ${ }^{11}$ However, recent innovations in cellular and molecular imaging methods have enabled the detection of key biological processes and vessel characteristics that may correlate with aneurysm disease progression. ${ }^{12-14}$

Superparamagnetic particles of iron oxide (SPIO) are part of a novel class of MRI contrast agents that provide additional biological and functional information through the detection of cellular inflammation within tissues. Current preparations of ultrasmall SPIO (USPIO) are biodegradable and safe for clinical administration. ${ }^{15-17}$ USPIO are phagocytosed by macrophages within vascular and lymphatic tissues ${ }^{18-21}$ and can be used to detect tissue inflammation. USPIO accumulate in ruptured and vulnerable carotid plaques, and USPIO-enhanced MRI can detect a reduction in plaque inflammation following treatment with high-dose atorvastatin. ${ }^{22}$ USPIO uptake has also been demonstrated in other regions of the vasculature, including the aorta, in preclinical models and also in small clinical studies. ${ }^{23} 24$

We have recently conducted a series of MRI studies in patients with AAA and shown that uptake of USPIO in the aortic wall correlates with macrophage activity, and identifies cellular inflammation. ${ }^{25}$ Using a $3 \mathrm{~T}$ MR scanner, patients with asymptomatic AAA ( $\mathrm{n}=29$; aneurysm diameter $4.0-6.6 \mathrm{~cm}$ ) attending our surveillance programme were imaged before and 24-36 h after intravenous administration of USPIO. Histological examination of aneurysm tissue confirmed co-localisation and uptake of USPIO in areas of macrophage infiltration.

Patients exhibiting USPIO uptake and inflammation within the wall of their aneurysm had a threefold higher aneurysm expansion rate $(\mathrm{n}=11,0.66 \mathrm{~cm} /$ year; $\mathrm{p}=0.020)$ than patients with no $(\mathrm{n}=6,0.22 \mathrm{~cm} / \mathrm{y})$ or non-specific $(\mathrm{n}=8,0.24 \mathrm{~cm} / \mathrm{y})$ USPIO uptake, despite similar baseline anteroposterior diameters. ${ }^{25}$ Indeed, one patient with a hotspot of USPIO uptake and biological activity within the wall of an aneurysm, died suddenly 2 months after scanning, from presumed aneurysm rupture.

We have shown that USPIO-enhanced MRI holds major promise as a new method of risk-stratification of patients with AAA and extends beyond simple anatomical measurements of aneurysm diameter. The aim of this present study is to validate this technique within a larger multicentre cohort study and to examine the relationship between baseline USPIO uptake and subsequent aneurysm expansion, requirement for elective repair and incidence of rupture.

\section{METHODS AND ANALYSIS Study design}

The study is a prospective observational multicentre cohort study of patients with AAA disease under routine clinical surveillance, and will assess the role of MRI with USPIO to predict clinical disease progression.

\section{Study objectives}

Our previous proof-of-concept study ${ }^{25}$ demonstrated that MRI with USPIO had the potential to predict clinical disease progression in patients with asymptomatic AAA. The aim of this study is to validate these findings, focusing on the relationship between baseline USPIO uptake and the subsequent clinical outcome and aneurysm expansion rates.

The primary objective of the study is to determine whether mural uptake of USPIO in AAA provides 
incremental risk prediction in addition to standard risk markers such as aneurysm diameter, smoking and blood pressure.

Secondary objectives of the study are to ascertain whether mural uptake of USPIO in AAA: (1) correlates with the rate of aneurysm expansion; (2) occurs more commonly in patients who progress to surgery or whose aneurysm subsequently ruptures; (3) co-localises with, or relates to, areas of biomechanical stress and (4) occurs in a reproducible manner.

We will also investigate other inter-related mechanisms associated with aneurysm growth. We will specifically explore the added value of biomechanical stress modelling as we suspect co-localisation of USPIO uptake and areas of high mechanical stress could act synergistically, and cause more marked aneurysm growth. We will also examine correlates with other blood biomarkers including regulators of extracellular matrix turnover (such as matrix metalloproteinases and tissue inhibitors of metalloproteinases) and vascular inflammation (such as $\mathrm{C}$ reactive protein and interleukin 6 ).

Reproducibility of the technique will be assessed in a subgroup $(n=20)$ of study participants (from the Edinburgh study centre) who will undergo repeat MRI and USPIO administration at $<1$ month and 1 year. Short-term $(<1$ month) reproducibility is likely to reflect the variability in USPIO uptake whereas long-term (1 year) reproducibility is likely to represent the variation in biology of the aortic aneurysm with time.

This will lay the foundation for a large multicentre randomised controlled trial targeted at applying this technique to determine clinical management and surgical intervention. This will be particularly important given the fact that screening programmes for the detection of AAA are being rolled out in many countries and this is likely to increase substantially the number of patients being entered into surveillance programmes.

\section{Study end points}

The primary endpoint of the study will be the composite rate of aneurysm rupture or repair. The following secondary endpoints will be evaluated: (1) the rate of aneurysm rupture; (2) the rate of surgical or endovascular repair of the aneurysm; (3) the aneurysm growth rate and (4) all-cause and aneurysm-related mortality.

We will conduct exploratory analyses examining the interactions between mural USPIO uptake, biomechanical stress, clinical risk factors, and serum biomarkers of extracellular matrix turnover and inflammation.

\section{Study population}

Participants will be identified from clinical surveillance programmes and databases at three centres: Royal Infirmary of Edinburgh, Western Infirmary in Glasgow and Forth Valley Royal Hospital (FVRH). We will recruit 350 participants over a 24-month period with recruitment having begun on 6 November 2012. Recruitment rate and summary statistics can be viewed at online supplementary appendix 2 .

Inclusion criteria will be: (1) AAA measuring $\geq 4.0 \mathrm{~cm}$ in anteroposterior diameter on ultrasound scanning and (2) age $\geq 40$ years. Patients under the age of 40 years will be excluded since they may have a connective tissue disorder accounting for their disease.

Exclusion criteria will be: (1) patients expected to undergo imminent elective or emergency surgical or endovascular repair; (2) contraindication to MRI scanning identified from MRI safety questionnaire; (3) patients refusing or unable to give informed consent; (4) women with childbearing potential or who are breast feeding will not be enrolled into the study (women who are currently pregnant or have experienced menarche but are premenopausal and have not been sterilised will be excluded); (5) intercurrent illness including patients with a systemic inflammatory disorder or underlying malignancy (life expectancy <2 years); (6) renal dysfunction (estimated glomerular filtration rate $\left.\leq 30 \mathrm{~mL} / \mathrm{min} / 1.73 \mathrm{~m}^{2}\right)$; (7) polycythaemia; (8) contraindication to ferumoxytol (evidence of known iron overload, haemochromatosis, known hypersensitivity to ferumoxytol or its components or anaemia not caused by iron deficiency) and (9) contraindication to iodine-based contrast.

\section{Participant selection and enrolment}

Eligible participants will be identified from the clinical aneurysm surveillance database and will be approached by a member of the study team at their ultrasound appointment. Participants will be supplied with the study information sheet either in person or by post. Written informed consent will be obtained from patients willing to participate in the study. Participants are free to withdraw from the study at any point or the investigator can choose to withdraw a participant. If a participant has consented for the study and then wishes to withdraw, providing they have not been administered ferumoxytol, another participant will be recruited in their place.

\section{Study drug}

Ferumoxytol

Ferumoxytol (Rienso) is composed of USPIO coated with polyglucose sorbitol carboxymethylether. It is supplied as an aqueous colloidal product that is formulated with mannitol and presented in single use vials ready for intravenous infusion. Each vial contains $510 \mathrm{mg}$ of elemental iron in a volume of $17 \mathrm{~mL}$ of mannitol.

The ferumoxytol dose $(4 \mathrm{mg} / \mathrm{kg})$ is removed from the vial and administered intravenously at a rate of up to $1 \mathrm{~mL} / \mathrm{s}$. The single dose is given immediately following the baseline MRI and 24-36 $\mathrm{h}$ before the postcontrast scan. Blood pressure is recorded before and $30 \mathrm{~min}$ after administration to monitor for hypotension. In a subset of patients $(\mathrm{n}=20)$, MRI scanning and USPIO administration will be repeated at $<1$ month and 1 year (up to a total of 3 doses of ferumoxytol in 1 year). Each 
dose of ferumoxytol equates to approximately $7 \%$ of total body iron.

The study drug is manufactured by AMAG Pharmaceuticals Inc (Lexington, Massachusetts, USA), and distributed and released by Takeda Italia Farmaceutica SPA in Europe.

\section{Buscopan}

Intravenous Buscopan (hyoscine butylbromide) $20 \mathrm{mg}$ will be administered before each MRI to reduce artefact from bowel peristalsis in patients who do not have a contraindication (allergic reaction, narrow angle glaucoma, paralytic ileus, myasthaenia gravis, obstructive prostatic hypertrophy).

\section{Study assessments and data collection}

Online supplementary appendix 1 outlines the assessments performed at each study visit. Figure 1 shows the study flow chart. Patients recruited in Edinburgh and Glasgow will undergo all study investigations in their respective centre. For patients recruited from FVRH, they will have the choice of attending the study in either Edinburgh or Glasgow. They will continue to have their surveillance ultrasound scan at FVRH.

\section{Clinical assessment}

Patients will have a full formal and standardised clinical assessment at baseline that will include history, examination and documentation of cardiovascular risk factor profile (smoking status, family history, hypertension, hyperlipidaemia, diabetes mellitus). Concomitant medications (antihypertensive medication, etc) will be recorded at baseline and at the end of the study.
Figure 1 Study flow chart (i.v., intravenous).

Patients with abdominal aortic aneurysms (identified in surveillance program and other study site clinics)

350 patients with abdominal aortic aneurysm $>40 \mathrm{~mm}$ who meet inclusion and exclusion criteria AND who agree to take part

\section{Baseline Assessment (2 days)}

Day 1:

- Consent

- Clinical Assessment

- Ultrasound scan (occurs as standard care and can $\propto$ cur up to 1 month prior to baseline assessment)

- 3T Magnetic resonance scan

- i.v. ferumoxytol

- Computed Tomography scan with contrast

- Blood sample collection (can be done on day 2)

- Pulse wave analysis (can be done on day 2)
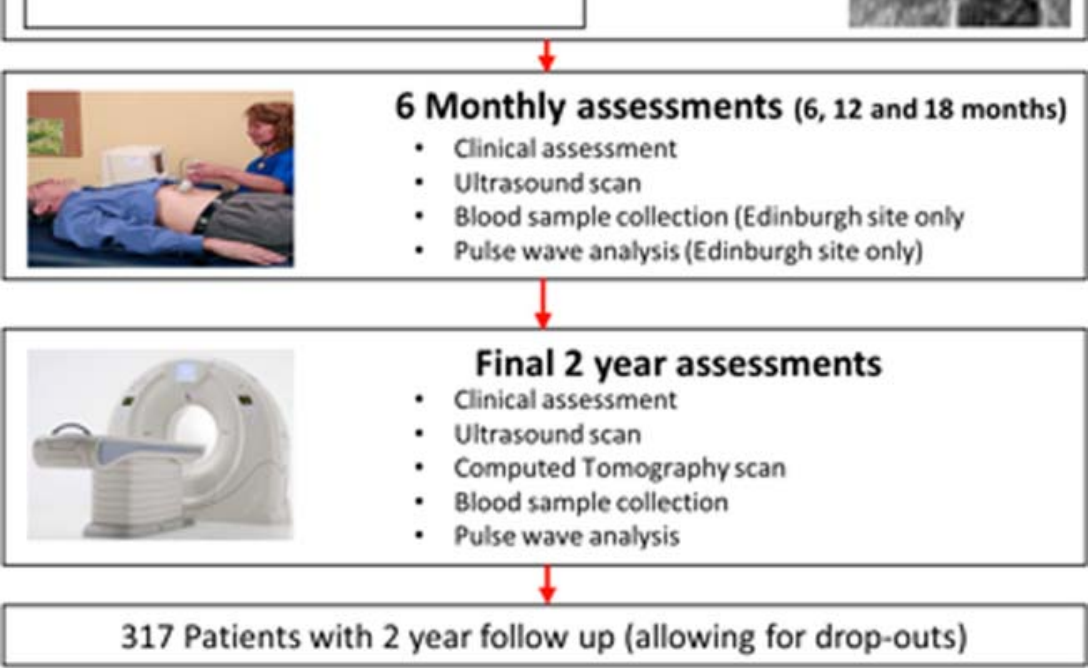

- 20 patients will undergo MRI reproducibility assessments

Day 2 (24-36 hours after Day 1):

- 3T Magnetic resonance scan (post ferumoxytol) *

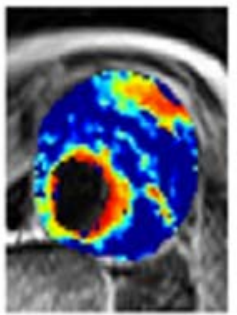


Blood sampling

Blood samples (20 mL on each visit) will be collected at baseline and at 24 months, for routine haematology and biochemistry including full blood count, urea, creatinine and electrolytes, liver function tests, total cholesterol and glucose.

For patients recruited and imaged in the Edinburgh centre, a further blood sample $(9 \mathrm{~mL})$ will be collected at baseline and at 6, 12, 18 and 24 months. Samples will be processed (plasma and serum) and stored at $-80^{\circ} \mathrm{C}$ for later analysis of potential extracellular matrix and inflammatory biomarkers.

\section{Pulse wave analysis and velocity}

Pulse wave analysis and velocity will be measured in triplicate in fasted patients using applanation tonometry (SphygmoCor, AtCor Medical, Sydney Australia). This will facilitate the measurement of central aortic pressure to assist in the modelling of biomechanical stress within the aneurysm. This will be measured at baseline and at $6,12,18$ and 24 months.

\section{Ultrasound of the abdominal aorta}

Patients will undergo ultrasound imaging as part of the standard clinical surveillance programme with measurement of the maximum anteroposterior diameter of the aneurysm. Ultrasound scans will be performed every 6 \pm 2 months. A $3.5 \mathrm{MHz}$ linear array transducer will be used to provide standard real time longitudinal B-scan images of the AAA at the point of maximum diameter. Maximum anteroposterior AAA diameter and distensibility (pressure strain elastic modulus and stiffness) will be assessed. Scans will be undertaken by accredited clinical vascular scientists with interobserver coefficient of variation of aortic diameter measurements of $3.5 \%$ in our laboratory. ${ }^{26}$

\section{CT of the abdominal aorta}

Contrast-enhanced images of the abdominal aorta will be obtained using a 320-multidetector (Edinburgh; Aquilion ONE; Toshiba) or 64-multidetector CT scanner (Glasgow; Brilliance 64; Philips), at baseline and 2 years. These data will be reconstructed into three dimensions (3D) using volumetric matrices to enable a more comprehensive assessment of the aneurysm geometry and growth than that provided by the ultrasound assessment of the unidimensional aortic anteroposterior diameter. In cases where study participants undergo emergency repair of a ruptured AAA, a CT scan may not be performed. In the event of patients not receiving the CT scan at baseline it will be performed within 1 month of the baseline visit.

\section{$\mathrm{MRI}$ of the abdominal aorta}

MRI will be conducted using a $3 \mathrm{~T}$ Siemens Magnetom Verio scanner (Siemens, Erlangen, Germany) before and $24-36 \mathrm{~h}$ after administration of the USPIO in Edinburgh or Glasgow. Scanning protocols from the two centres were validated prior to study initiation. The MRI safety questionnaire will be applied to identify patients with a contraindication to scanning. Patients will be given intravenous Buscopan (hyoscine butylbromide) prior to imaging to minimise bowel motion artefacts. Routine clinical coronal and sagittal breath-held T2-weighted (T2W) multislice HASTE sequences will be used to identify the position and extent of the aneurysm, following which a respiratory-gated, T2W turbo spin echo sequence will be used to acquire detailed anatomical data (TR/TE 2500/252 ms; matrix 365×384; field of view $300 \times 400 \mathrm{~mm}$; slice width $5 \mathrm{~mm}$ ). These data are acquired with and without Spectral Attenuated Inversion Recovery fat suppression in order to allow segmentation of aortic wall. A multiecho, gradient echo $\mathrm{T} 2 * \mathrm{~W}$ sequence (TE 4.9, 7.7, 10.5, $13.3 \mathrm{~ms}$; TR $133 \mathrm{~ms}$; flip angle $15^{\circ}$; matrix $192 \times 256$; field of view $400 \times 400 \mathrm{~mm}$; slice width $5 \mathrm{~mm}$ ) will be used to acquire contiguous axial images of the entire aneurysm (from the neck of the aneurysm down to the iliac bifurcation) with slice positions corresponding to those of the T2W images. Saturation bands are placed over the anterior abdomen for the $\mathrm{T} 2 * \mathrm{~W}$ acquisition in order to minimise breathing-related artefact.

\section{Clinical management of AAA}

The management of AAA in NHS Scotland follows international ${ }^{27}$ and national guidelines. ${ }^{28}$ Elective AAA repair is considered when the anteroposterior diameter, as measured by ultrasound scanning, reaches $5.5 \mathrm{~cm}$, the AAA grows $>1 \mathrm{~cm}$ in 1 year or the AAA is symptomatic. Furthermore, the decision for elective AAA repair will be made by independent clinicians who will be unaware of the USPIO-enhanced MRI. These clinicians do not have routine access to the CT scans. However, clinically important incidental findings will be communicated to the clinicians. Study investigators will not be directly involved in the patient's care or treatment decisions. In the case of patients undergoing surgery where repair of the AAA is not the primary intention (eg, in the case of repair of a common iliac aneurysm with concomitant AAA), they will be included in the primary analysis on an intention-to-treat analysis.

\section{Follow-up for patients in certain circumstances}

If a patient undergoes an open or endovascular repair of their aneurysm, we will record the results of any scans that are carried out for clinical reasons (such as extra CT or ultrasound scans). Those undergoing open repair will have aneurysm wall tissue collected and stored for the assessment of tissue resident macrophages and matrix metalloproteinases. These patients will be invited to return every 6 months for blood tests but not ultrasound or pulse wave analysis or velocity. 


\section{Proposed analysis}

Image analysis of ultrasound

Cursors will be locked onto echoes representing the anterior and posterior aortic walls and the movement of both tracked with each cardiac cycle. The longitudinal image will be used for analysis as experience with the technique and data from formal reproducibility studies indicate that the quality of the data obtained from a longitudinal section is superior to that obtained from a transverse view. Images will be captured digitally for later offline core laboratory review.

\section{Image analysis of CT}

CT of the abdominal aorta will be performed at baseline and 2 years to assess regional and volumetric growth rates. These two CT scans will be spatially co-registered so that areas of expansion and growth can be more accurately defined, and can permit correlation with areas of USPIO uptake and finite element analysis modelling.

Three-dimensional computer models will be generated from segmented, contrast-enhanced images, allowing geometric indices to be quantified. Semiautomatic segmentation is achievable due to the intensity differences between the aneurysm, and surrounding tissues and structures. Intensity thresholding will be used to identify the lumen. To detect the thrombus and complete the segmentation, we will use a 3D deformable model approach that utilises the level set algorithm as well as modelling the thrombus contour as a radial function starting from the aortic centre line as calculated from the lumen segmentation.

\section{Image analysis of MRI}

A region of interest encompassing the aortic wall and thrombus but excluding the lumen will be drawn on each slice of the precontrast T2W image. The precontrast T2W scan will be used as the base image to which subsequent scans will be co-registered using a semiautomatic rigid 3D voxel registration protocol utilising a normalised mutual information algorithm. To minimise the contribution of bowel and abdominal wall motion in the MRI datasets, a region for registration will include the aorta, vertebrae and spinal musculature that remain relatively static during the respiratory cycle, but exclude the more mobile abdominal wall and bowel.

The four echoes in the multiecho $\mathrm{T} 2 * \mathrm{~W}$ sequence will be combined to generate $\mathrm{T} 2 *$ and $\mathrm{R} 2 *$ maps. The $\mathrm{T} 2 *$ value is the decay constant for the exponential decay of signal intensity with time and its inverse is the $\mathrm{R} 2 *$ value. A $3 \times 3$ voxel Gaussian filter will be applied to the individual echoes to reduce noise, and the coefficient of determination will be used to exclude data that do not have an acceptable straight line fit when the log of signal intensity is plotted against echo time. Images will be co-registered to allow direct comparison of the precontrast and postcontrast T2*W MRI. USPIO uptake will be presented visually as a colour map of change in $\mathrm{T} 2 *$ or
R2* values between pre-USPIO and post-USPIO scans, with our previously validated threshold of significance applied (figure 2) ${ }^{25}$ The change in $\mathrm{T} 2 *$ or $\mathrm{R} 2 *$ colour maps from the MRI will be spatially co-registered to the CT datasets.

\section{Finite element analysis}

Using Abaqus/Complete Abaqus Environment, the vessel wall will be modelled as a constant thickness layer of $1.5 \mathrm{~mm}$, with a hyperelastic constitutive model. Thrombus will be modelled as homogeneous and isotropic with Young's modulus $0.1 \mathrm{MPa}$. The model will be inflated from diastolic to systolic pressure using a homogeneous pressure-modelling regime. Peak wall stress and other rupture indices will be calculated and compared against areas of USPIO uptake.

\section{Statistics and data analysis}

Sample size calculation

The sample size is determined by the numbers required to build robust prognostic models for rupture or surgical

\section{$\% \Delta$ in $\mathrm{T} 2 *$ value}

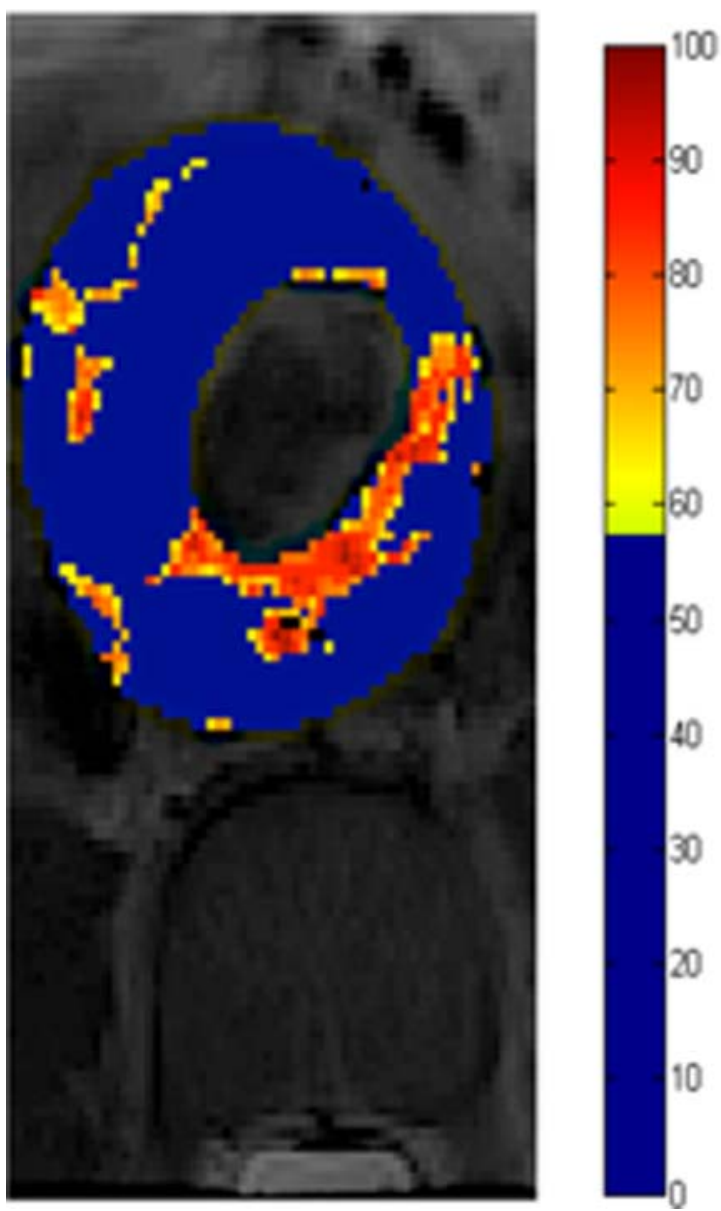

Figure 2 Colour map of MRI of an abdominal aortic aneurysm. Red and yellow pixels indicate areas of increased $\mathrm{T}^{*}$ value, indicative of ultrasmall superparamagnetic particles of iron oxide uptake. 
repair, and in particular to measure the additional prognostic value of mural USPIO uptake when added to such a prognostic model based only on conventional clinical risk factors. There is a widely accepted 'rule of thumb' that one needs at least 10 and ideally 20 outcome events per covariate to be included in a prognostic model. ${ }^{29}$ As described in more detail below, we plan to use the net reclassification index (NRI) as the primary measure of the clinical relevance of the added prognostic value of mural USPIO uptake. ${ }^{30}{ }^{31}$ Based on two recent papers using the NRI, ${ }^{32}{ }^{33}$ and also taking account of the Harrell 'rule of thumb', we estimate that we need to observe 130 events (ie, the composite of rupture or surgical repair) to have adequate sensitivity to answer the primary question. With our estimated event rate of $41 \%$ over the mean duration of follow-up of 2 years, ${ }^{34}$ this equates to 317 patients. The estimated event rate is based on a composite endpoint of rupture and repair. This is taken from a previous study at our centre, ${ }^{34}$ where rupture rates were $13 \%$ and elective intervention rates were in the region of $30 \%$ over a 2-year period.

The sample size has been derived so that the study will allow us to assess the 'added value' of USPIO uptake over and above conventional clinical risk factors in predicting rupture and/or the need for surgical intervention. As such, conventional type I and II errors are not relevant. However, the approach we have adopted is extremely conservative in terms of a conventional power calculation. For example, if we assume that $40 \%$ of the patients are 'positive' for USPIO uptake, and that their event rate is $55 \%$, compared with an event rate of $30 \%$ in the uptake 'negative' patients, then the statistical power to detect such an effect would be over $99 \%$ at the $5 \%$ significance level (2-sided). In other words, the study has extremely high power to detect an effect of the order that would be required if USPIO uptake is to add usefully to conventional prognostic factors.

There should be very modest losses to follow-up for the primary analysis, since all study recruits are already enrolled in a surveillance programme. Moreover, we shall seek consent at the time of recruitment to flag the patients so that if any patients are lost to follow-up they can be traced to identify any hospital admissions or to identify if they have died. In addition, some patients will drop out due to technical failure of the scan, such as claustrophobia or a severe reduction in image quality preventing analysis. Image quality will be assessed on a four-point Likert scale (1=excellent; $2=$ mild reduction in image quality; $3=$ moderate reduction in image quality; and $4=$ severe reduction in image quality). We have, therefore, conservatively accounted for a $10 \%$ drop out rate and we will recruit 350 patients to the study.

\section{Statistical analysis}

The baseline assessment will include the baseline aneurysm diameter, sex, smoking habit and blood pressure. Using these covariates, a prognostic model predicting the time to the composite outcome event of rupture or surgical repair will be developed using Cox proportional hazards regression. The added prognostic value of adding mural USPIO uptake to this model will be assessed using the increase in the area under the receiver operator characteristic curve, the Nagelkerke partial $\mathrm{R}^{2}$ and, primarily, the NRI. ${ }^{32} 33$ The NRI is a direct measure of the clinical relevance of adding a covariate to a prognostic model. It is an overall measure of how many patients move from a low predicted risk to a high predicted risk when the covariate is added (and whether these individuals are indeed high risk), and how many move from a high predicted risk to a low predicted risk (and whether these individuals are indeed low risk). For the primary analysis, the USPIO will be taken as a binary covariate (as in Richards $e t a l^{25}$ ), but a sensitivity analysis will explore the potential for including a quantification of the USPIO uptake. Some but not all of the patients will also have a measure of current aneurysm growth rate at baseline, and a further sensitivity analysis will explore using growth rate as a further prognostic factor, using imputation when the estimated growth rate is not available.

A secondary analysis will follow a similar analytical strategy to that set out above, but using prognostic models to predict aneurysm growth rate. This analysis will be far more complex with the inclusion of serial measures of aneurysm diameter and with these measurements being censored on rupture or on surgical repair. Further exploratory analysis will look at the predictive ability of measures of wall stress and how these interact with regional USPIO uptake.

The reproducibility of the technique will be assessed with participants categorised with respect to the presence or absence of USPIO uptake at 1 month and 1 year, to define the proportionate agreement, with $95 \%$ CIs.

\section{ETHICS AND DISSEMINATION}

Research sponsorship is provided by the Academic and Clinical Central Office for Research and Development (ACCORD), a partnership between the University of Edinburgh and NHS Lothian Health Board.

The results of the study will be submitted for publication in peer-reviewed journals.

Results will also be disseminated through presentations at national and international conferences.

\section{DISCUSSION}

The multicentre $\mathrm{MA}^{3} \mathrm{RS}$ study will assess the ability of USPIO-enhanced MRI to augment standard risk markers for AAA expansion or rupture in 350 patients attending aneurysm surveillance clinics. This is the first study to assess novel MRI-based imaging approaches to improve risk prediction in this common and potentially fatal condition.

Mortality from ruptured AAA is very high, at approximately $90 \%$. In contrast, the risk of death from open 
surgical or endovascular repair is low, at $1-5 \% .^{35}$ The aim is therefore to identify AAA prior to rupture and consider surgery when the risk of rupture exceeds that of elective repair. Aneurysm screening reduces the risk of aneurysm-related death by $53-73 \%$, and has been introduced throughout the UK and in many countries across the world. ${ }^{136}{ }^{37}$ Evaluation of rupture risk is currently based on the maximum anteroposterior diameter of the aneurysm. Elective aneurysm repair is offered to patients with an aneurysm diameter of $>5.5 \mathrm{~cm}$ or if aneurysm expansion is $>1.0 \mathrm{~cm} /$ year. However, while the risk of rupture increases with diameter, ${ }^{8} 3839$ the absolute diameter of the aneurysm is not the sole determinant of the risk of rupture, ${ }^{40}$ and elective repair when the diameter is $<5.5 \mathrm{~cm}$ offers no survival benefit over continued surveillance. ${ }^{41}$ Furthermore, 1 in 14 screendetected aneurysms $<5.5 \mathrm{~cm}$ in diameter will rupture within 3 years of detection, and up to one-fifth of ruptured AAA are $<5.5 \mathrm{~cm}$ in diameter, while many patients present with diameters considerably $>5.5 \mathrm{~cm}$ without prior symptoms or rupture. ${ }^{8} 4142$ The increase in patients under long-term surveillance with the introduction of nationwide screening, coupled with the unpredictability and non-linearity of aneurysm growth, highlight the need for an improved method of risk prediction in aneurysm disease. This will then enable preventative surgical or endovascular repair to be targeted to the high-risk patients.

We have previously demonstrated that the assessment of USPIO uptake with MRI can be used to detect focal hotspots of inflammation in asymptomatic aneurysms, and that mural USPIO uptake is associated with more rapid expansion of aneurysms. ${ }^{25}$ By assessing these biological processes and evaluating them with this novel technique, the $\mathrm{MA}^{3} \mathrm{RS}$ study aims to develop an improved method of assessing rupture risk in AAA in a large prospective clinical study with a long duration of follow-up.

We will model differing thresholds of risk predictors that have the potential to inform future interventional trials. For example, this study could define USPIOenhanced MRI features that predict aneurysm rupture. This could lead to the design of a major interventional trial whereby surgical intervention could be targeted at those aneurysms that are vulnerable or prone to rupture. This could include surgery in patients with aneurysms $<5.5 \mathrm{~cm}$ or mandate conservative management in those with aneurysms $>5.5 \mathrm{~cm}$. A more sophisticated stratified approach has the potential to save lives as well as avoid unnecessary high-risk surgery in those who are unlikely to come to harm.

\section{Trial status}

The study has been approved by the East of Scotland Research Ethics Committee. Recruitment is underway at all sites and two-thirds of the study population have already been recruited. Completion of study enrolment is anticipated in autumn 2014.
Author affiliations

${ }^{1}$ British Heart Foundation/University of Edinburgh Centre for Cardiovascular Science, Edinburgh, UK

${ }^{2}$ BHF Glasgow Cardiovascular Research Centre, University of Glasgow, Glasgow, UK

${ }^{3}$ Forth Valley Royal Hospital, Larbert, UK

Collaborators $M A^{3} R S$ Trialists: Chief Investigator: DEN. Principal Investigators Edinburgh: OMBM, RF and AV. Principal Investigator Glasgow: WS. Principal Investigator Forth Valley Royal Hospital: RH. Grant applicants: DEN, GMc, RTAC, PB, JMJR, SS, TJM, CG, OJG, PH, BD, EJRvB, GMu, CB and WS. Trial Steering Committee: Professor Julie Brittenden (Chair), Professor Graeme Houston, Robert Lambie, Professor John Norrie, DEN, GMc, SS, PB, GMu, KG, KO and OMBM. Edinburgh Clinical Trials Unit. GMu, KG, KO, Lynsey Milne, Garry Milne and CG.

Contributors DEN and JMJR conceived the study, participated in its design and coordination, and helped draft the manuscript. OMBM helped to draft the manuscript. All authors read and approved the final manuscript.

Funding The study was funded by a grant from the Medical Research Council National Institute for Health Research Efficacy and Mechanism Evaluation (NIHR EME) programme. Additional contributions came from the Chief Scientists Office (CSO; ETM/365). DEN is supported by the British Heart Foundation (CH/09/002) and the Wellcome Trust (WT103782AIA). OMBM is supported by the Academic Department of Military Surgery and Trauma. The Clinical Research Imaging Centre and Wellcome Trust Clinical Research Facility (Edinburgh), and the Clinical Research Facility Glasgow, are supported by National Health Service Research Scotland (NRS). The Glasgow Cardiovascular Research Centre is supported by the British Heart Foundation.

Competing interests None.

Ethics approval East of Scotland Research Ethics Committee (A).

Provenance and peer review Not commissioned; externally peer reviewed.

Data sharing statement No additional data are available.

Open Access This is an Open Access article distributed in accordance with the Creative Commons Attribution Non Commercial (CC BY-NC 4.0) license, which permits others to distribute, remix, adapt, build upon this work noncommercially, and license their derivative works on different terms, provided the original work is properly cited and the use is non-commercial. See: http:// creativecommons.org/licenses/by-nc/4.0/

\section{REFERENCES}

1. Ashton HA, Buxton MJ, Day NE, et al., Multicentre Aneurysm Screening Study Group. The Multicentre Aneurysm Screening Study (MASS) into the effect of abdominal aortic aneurysm screening on mortality in men: a randomised controlled trial. Lancet 2002;360:1531-9.

2. Gillum RF. Epidemiology of aortic aneurysm in the United States. J Clin Epidemiol 1995;48:1289-98.

3. Logan AJ, Bourantas NI, Moseley LG, et al. Mortality from ruptured abdominal aortic aneurysm in Wales. Br J Surg 2000;87:966-7.

4. Johnston KW. Ruptured abdominal aortic aneurysm: six-year follow-up results of a multicenter prospective study. Canadian Society for Vascular Surgery Aneurysm Study Group. J Vasc Surg 1994;19:888-900.

5. Brady AR, Thompson SG, Fowkes FG, et al. Abdominal aortic aneurysm expansion: risk factors and time intervals for surveillance. Circulation 2004;110:16-21.

6. Raghavan ML, Vorp DA. Toward a biomechanical tool to evaluate rupture potential of abdominal aortic aneurysm: identification of a finite strain constitutive model and evaluation of its applicability. J Biomech 2000;33:475-82.

7. Vallabhaneni SR, Gilling-Smith GL, How TV, et al. Heterogeneity of tensile strength and matrix metalloproteinase activity in the wall of abdominal aortic aneurysms. J Endovasc Ther 2004;11:494-502.

8. Thompson AR, Cooper JA, Ashton HA, et al. Growth rates of small abdominal aortic aneurysms correlate with clinical events. Br J Surg 2009;97:37-44.

9. Brown PM, Zelt DT, Sobolev B. The risk of rupture in untreated aneurysms: the impact of size, gender, and expansion rate. $J$ Vasc Surg 2003;37:280-4. 
10. Cai J, Hatsukami TS, Ferguson MS, et al. In vivo quantitative measurement of intact fibrous cap and lipid-rich necrotic core size in atherosclerotic carotid plaque: comparison of high-resolution, contrast-enhanced magnetic resonance imaging and histology. Circulation 2005;112:3437-44.

11. Kramer CM, Cerilli LA, Hagspiel K, et al. Magnetic resonance imaging identifies the fibrous cap in atherosclerotic abdominal aortic aneurysm. Circulation 2004;109:1016-21.

12. Jaffer FA, Libby P, Weissleder R. Molecular imaging of cardiovascular disease. Circulation 2007:116:1052-61.

13. Rajani N, Joshi FR, Tarkin JM, et al. Advances in imaging vascular inflammation. Clin Transl Imaging 2013;1:305-14.

14. Choudhury RP, Fisher EA. Molecular imaging in atherosclerosis, thrombosis, and vascular inflammation. Arterioscler Thromb Vasc Biol 2009;29:983-91.

15. Bernd H, De Kerviler E, Gaillard S, et al. Safety and tolerability of ultrasmall superparamagnetic iron oxide contrast agent: comprehensive analysis of a clinical development program. Invest Radiol 2009;44:336-42.

16. Bourrinet $\mathrm{P}$, Bengele $\mathrm{HH}$, Bonnemain $\mathrm{B}$, et al. Preclinical safety and pharmacokinetic profile of ferumoxtran-10, an ultrasmall superparamagnetic iron oxide magnetic resonance contrast agent Invest Radiol 2006;41:313-24.

17. Müller K, Skepper JN, Posfai M, et al. Effect of ultrasmall superparamagnetic iron oxide nanoparticles (Ferumoxtran-10) on human monocyte-macrophages in vitro. Biomaterials 2007;28:1629-42.

18. Harisinghani MG, Barentsz J, Hahn PF, et al. Noninvasive detection of clinically occult lymph-node metastases in prostate cancer. $N$ Engl J Med 2003;348:2491-9.

19. Heesakkers R, Hövels AM, Jager GJ, et al. MRI with a lymph-node-specific contrast agent as an alternative to CT scan and lymph-node dissection in patients with prostate cancer: a prospective multicohort study. Lancet Oncol 2008;9:850-6.

20. Kooi ME, Cappendijk VC, Cleutjens KBJM, et al. Accumulation of ultrasmall superparamagnetic particles of iron oxide in human atherosclerotic plaques can be detected by in vivo magnetic resonance imaging. Circulation 2003;107:2453-8.

21. Trivedi RA, Mallawarachi C, U-King-Im J-M, et al. Identifying inflamed carotid plaques using in vivo USPIO-enhanced MR imaging to label plaque macrophages. Arterioscler Thromb Vasc Biol 2006;26:1601-6.

22. Tang TY, Howarth SPS, Miller SR, et al. The ATHEROMA (Atorvastatin Therapy: Effects on Reduction of Macrophage Activity) Study. Evaluation using ultrasmall superparamagnetic iron oxide-enhanced magnetic resonance imaging in carotid disease. Journal Am Coll Cardio 2009;53:2039-50.

23. Sadat U, Taviani V, Patterson AJ, et al. Ultrasmall superparamagnetic iron oxide-enhanced magnetic resonance imaging of abdominal aortic aneurysms-a feasibility study. Eur $J$ Vasc Endovasc Surg 2011;41:167-74

24. Turner GH, Olzinski AR, Bernard RE, et al. Assessment of macrophage infiltration in a Murine model of abdominal aortic aneurysm. J Magn Reson Imaging 2009;30:455-60.
25. Richards JMJ, Semple SI, MacGillivray TJ, et al. Abdominal aortic aneurysm growth predicted by uptake of ultrasmal superparamagnetic particles of iron oxide: a pilot Study. Circ Cardiovasc Imaging 2011:4:274-81.

26. Wilson KA, Hoskins PR, Lee AJ, et al. Ultrasonic measurement of abdominal aortic aneurysm wall compliance: a reproducibility study. J Vasc Surg 2000;31:507-13.

27. Hirsch AT, Haskal ZJ, Hertzer NR, et al. ACC/AHA 2005 practice guidelines for the management of patients with peripheral arterial disease (lower extremity, renal, mesenteric, and abdominal aortic). Circulation 2006;113:e463-5

28. Nice.org.Uk (http://www.nice.org.uk)

29. Harrell FE, Lee KL, Califf RM, et al. Regression modelling strategies for improved prognostic prediction. Stat Med 1984;3:143-52.

30. Pencina MJ, D'Agostino RB, D'Agostino RB, et al. Evaluating the added predictive ability of a new marker: from area under the ROC curve to reclassification and beyond. Stat Med 2008;27:157-72; discussion 207-12.

31. Pencina MJ, D'Agostino RB, Steyerberg EW. Extensions of net reclassification improvement calculations to measure usefulness of new biomarkers. Stat Med 2011;30:11-21.

32. Fellahi J-L, Le Manach Y, Daccache G, et al. Combination of EuroSCORE and cardiac troponin I improves the prediction of adverse outcome after cardiac surgery. Anesthesiology 2011;114:330-9.

33. Polak JF, Pencina MJ, Pencina KM, et al. Carotid-wall intima-media thickness and cardiovascular events. N Engl J Med 2011;365:213-21.

34. Wilson KA, Lee AJ, Lee AJ, et al. The relationship between aortic wall distensibility and rupture of infrarenal abdominal aortic aneurysm. J Vasc Surg 2003;37:112-17.

35. National Vascular Registry. 2013 Report on Surgical Outcomes Consultant-level statistics. 2013:1-62.

36. Fleming C, Whitlock EP, Beil TL, et al. Screening for abdominal aortic aneurysm: a best-evidence systematic review for the U.S. Preventive Services Task Force. Ann Intern Med 2005;142:203-11.

37. Lindholt JS, Juul S, Fasting $\mathrm{H}$, et al. Screening for abdominal aortic aneurysms: single centre randomised controlled trial. $B M J$ 2005;330:750.

38. Mofidi R, Goldie VJ, Kelman J, et al. Influence of sex on expansion rate of abdominal aortic aneurysms. Br J Surg 2007;94:310-14.

39. Vorp DA, Vande Geest JP. Biomechanical determinants of abdominal aortic aneurysm rupture. Arterioscler Thromb Vasc Biol 2005;25:1558-66.

40. Darling RC, Messina CR, Brewster DC, et al. Autopsy study of unoperated abdominal aortic aneurysms. The case for early resection. Circulation 1977;56:II161-4.

41. Greenhalgh RM, Brady AR, Brown LC, et al. Mortality results for randomised controlled trial of early elective surgery or ultrasonographic surveillance for small abdominal aortic aneurysms. The UK Small Aneurysm Trial Participants. Lancet 2005;352:1649-55.

42. Nicholls SC, Gardner JB, Meissner MH, et al. Rupture in small abdominal aortic aneurysms. J Vasc Surg 1998;28:884-8. 